\title{
Guest Editorial: Special Issue on Theoretical Aspects of Computer Science
}

\author{
Heribert Vollmer ${ }^{1} \cdot$ Brigitte Vallée $^{2}$
}

Published online: 28 June 2019

(C) Springer Science+Business Media, LLC, part of Springer Nature 2019

This special issue contains seven articles which are based on extended abstracts that were presented at the 34th Symposium on Theoretical Aspects of Computer Science (STACS), which was held at the Leibnizhaus in Hannover from March 8th to 11th, 2017. These extended abstracts were chosen among the top papers of those that were selected for presentation at STACS 2017 in a highly competitive peer-review process (after which only 54 papers out of 212 submissions were accepted).

Compared with the original extended abstracts, the articles have been extended with a description of the context, full proofs and additional results. They underwent a further rigorous reviewing process, following the TOCS Journal standard, completely independent from the selection process of STACS 2017.

The topics of the chosen papers cover combinatorics on words, circuit complexity, computational geometry, (parameterized) algorithms for graph problems, Markov chains, and stochastic games.

The article On Long Words Avoiding Zimin Patterns by Arnaud Carayol and Stefan Göller extends a classical result on combinatorics on words concerning unavoidable patterns, so called Zimin patterns. The authors study the question of the smallest length (depending on alphabet size) of a word that guarantees that a certain pattern appears - a very active research area in recent years. The main contribution here is a non-elementary lower bound on some of these minimal lengths.

A current focal issue in computational complexity is the study of size and depth of Boolean circuits computing the majority function or a nested majority function. The

This article is part of the Topical Collection on Special Issue on Theoretical Aspects of Computer Science (STACS 2017)

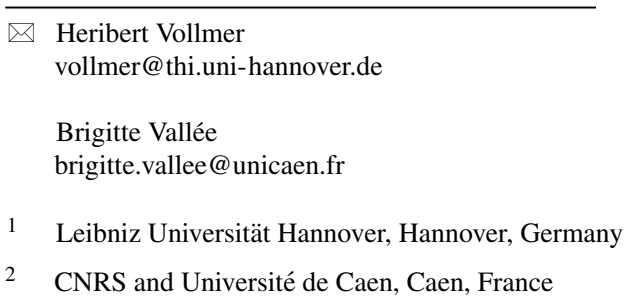


article Computing Majority by Constant Depth Majority Circuits with Low Fan-in Gates by Alexander Kulikov and Vladimir Podolskii asks for which $k$ the majority of $n$ Boolean inputs can be computed by depth 2 circuits consisting of gates computing majority of $k$ inputs (independent of circuit size). Deep proofs lead to new upper and lower bounds on this problem.

The article Counting Edge-injective Homomorphisms and Matchings on Restricted Graph Classes by Radu Curticapean, Holger Dell, and Marc Roth addresses a counting problem in graph theory, namely to determine the number of all matchings with exactly $k$ edges in a given input graph. If $k$ is considered as parameter, this problem is known to be \# W[1]-hard. It is proven that the problem remains hard even for very restricted graph classes such as line graphs or bipartite graphs with degree 2 on one side.

The article Complete Semialgebraic Invariant Synthesis for the Kannan-Lipton Orbit Problem by Nathanaël Fijalkow, Pierre Ohlmann, Joël Ouaknine, Amaury Pouly and James Worrell deals with the Orbit Problem: given a matrix $A$ together with vectors $x$ and $y$ on $\mathbb{Q}^{d}$, determine whether the orbit of $x$ under iterations of $A$ can ever reach $y$. This problem was famously shown to be decidable by Kannan and Lipton in the 1980's. In this paper, the authors wish to synthesise suitable invariants that are stable under $A$ and contain $x$ but not $y$. Such sets provide compact and versatile certificates of non-reachability. The authors show that whether a given instance of the Orbit Problem admits a semialgebraic invariant is decidable, and moreover, in positive instances, they provide an algorithm to synthesise suitable succinct invariants of polynomial size.

The article Improved Distance Queries and Cycle Counting by Frobenius Normal Form by Piotr Sankowski, and Karol Wegrzycki is devoted to counting cycles and walks in a unweighted directed graph in time $\tilde{O}\left(n^{\omega}\right)$ where $\omega$ is the exponent of square matrix multiplication. The method is based on the fast decomposition into Frobenius normal form and the Hankel matrix-vector multiplication. It allows the authors to solve the All-Nodes Shortest Cycles, All-Pairs All Walks problems efficiently and also give some improvement upon distance queries in unweighted graphs.

The article Mixing Time for Some Adjacent Transposition Markov Chains by Shahrzad Haddadan and Peter Winkler studies mixing time of certain Markov chains on the set $S_{n}$ of permutations on $\{1,2, \ldots, n\}$ in which adjacent transpositions are made with probabilities that depend on the items being transposed. One case of particular interest is what is called the "gladiator chain", in which each number $g$ is assigned a weight $s_{g}$ and when $g$ and $g^{\prime}$ are adjacent and chosen for possible swapping, $g$ comes out on top with probability $s_{g} /\left(s_{g}+s_{g^{\prime}}\right)$. The authors obtain a polynomial-time upper bound on mixing time in the particular case where there are only three weight classes.

The article The operator approach to entropy games by Marianne Akian, Stéphane Gaubert, Julien Grand-Clément, Jérémie Guillaud, studies a class of matrix multiplication games introduced at STACS'2016, named entropy games. One player, called "Despot", wishes to minimize the Perron root of a matrix, measuring the "freedom" of a half-Player, called "People". The opponent of Despot, called "Tribune", wishes to maximize this freedom. In the present paper, the authors show that entropy games 
are equivalent to a class of mean payoff games with perfect information, in which actions are elements of simplices and payments are given by relative entropies. This allows them to develop an "operator approach" for entropy games, deriving properties of the game and establishing complexity results as consequences of minimality properties of the Shapley operator (one-day dynamic programming operator) of the game.

We thank the authors for submitting their papers to this issue, and we thank the referees for their thorough reviews of the manuscripts.

Heribert Vollmer and Brigitte Vallée, Guest Editors, April 2019

Publisher's Note Springer Nature remains neutral with regard to jurisdictional claims in published maps and institutional affiliations. 